\title{
Oxidation of 3,4-dialkyl substituted isothiazolium salts to 1,1-dioxides
}

\author{
Antje Noack, Janine Wolf and Bärbel Schulze* \\ Institute of Organic Chemistry, University of Leipzig, Johannisallee 29, \\ D-04103 Leipzig, Germany \\ E-mail: bschulze@,chemie.uni-leipzig.de
}

Dedicated to Professor Heinz Heimgartner on the occasion of his $70^{\text {th }}$ birthday

\begin{abstract}
A new approach to 2-aryl-3-methylene-2,3-dihydroisothiazole 1,1-dioxides 11 from 3,4-dialkyl substituted $N$-phenyl-isothiazolium salts 6 via stable hydroperoxides 7 by reduction and elimination of water is introduced. Furthermore, 2-aryl-3-methylene-2,3-dihydroisothiazole 1,1-dioxides $\mathbf{1 1}$ are shown to be interesting synthons for the synthesis of 3-oxyfunctionalized sultams 8 and 9.
\end{abstract}

Keywords: Oxidations, hydroperoxides, sultams, methylene substituted isothiazoles

\section{Introduction}

Functionalized monocyclic sultams are known in the recent years to attract much attention due to their importance in biological and pharmaceutical research. ${ }^{1}$ The first monocyclic 2,3-dihydroisothiazole 1,1-dioxide $1\left(\mathrm{R}^{1}=\mathrm{NH}_{2}\right)$ with anti-HIV-1 activity has recently been prepared. ${ }^{1-3}$ The sultams 1 can be used as fungicides, herbicides and pesticides. ${ }^{4}$ The synthesis of such cyclic vinylsultams 2 by ring-closing metathesis (RCM) of vinylsulfonamide templates in the presence of Grubbs catalyst has been described. ${ }^{5}$

Sultams 3 react with cyclopentadiene in stereoselective Diels-Alder reactions to yield endonorbornenyl sulfonamides as the major diastereomer. Ring-opening metathesis polymerization (ROMP) of these cycloadducts was applied to prepare oligomeric sulfonamides (Scheme 1) .6,7 
<smiles>[R]C1=C(c2ccccc2)S(=O)(=O)N([R])C1([R])[R]</smiles>

1

$$
\begin{aligned}
& \mathrm{R}=\mathrm{H}, \mathrm{Me}, \mathrm{t}-\mathrm{Bu} \\
& \mathrm{R}^{1}=\mathrm{NH}_{2}, \mathrm{OH} \\
& \mathrm{R}^{3}=\mathrm{H}, \mathrm{Me}, \mathrm{Bz}
\end{aligned}
$$

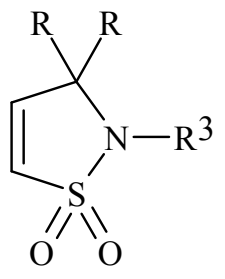

2

$$
\begin{aligned}
& \mathrm{R}=\mathrm{H}, \mathrm{Me}, \mathrm{t}-\mathrm{Bu} \\
& \mathrm{R}^{3}=\mathrm{H}, \mathrm{Me}, \mathrm{Bz}
\end{aligned}
$$<smiles>[R]C1=C([R])S(=O)(=O)N([R])C1=O</smiles>

3

$\mathrm{R}^{1}=\mathrm{R}^{2}=\mathrm{H}, \mathrm{Me}$

$\mathrm{R}^{3}=\mathrm{H}, \mathrm{t}-\mathrm{Bu}, \mathrm{CH}_{2} \mathrm{COOEt}$

Scheme 1. Functionalized monocyclic sultams 1-3.

Recently we prepared a series of 3-hydroperoxy-, hydroxy- as well as alkoxyderivatives by oxyfunctionalization of monocyclic isothiazolium salts $4\left(\mathrm{R}^{1}=\mathrm{CH}_{3}\right)$ which are unsubstituted at the 3-position. ${ }^{8,9}$ Here we report a new approach to introduce the oxy substituents at the methyl substituted 3-position of the isothiazole ring 6 with simultaneous oxidation of the sulfur to the sulfur dioxide in a one-step process. Furthermore we were able to compare now different pathways to prepare oxyfunctionalized 3-methyl-2,3-dihydroisothiazole 1,1-dioxides.

\section{Results and Discussion}

3-Methylisothiazolium salts $\mathbf{6}$, used as starting compounds for the following reactions, were synthesized by ring transformation of 3-unsubstituted isothiazolium salts 4 with anilines 5 by a known method (Scheme 2). ${ }^{10}$

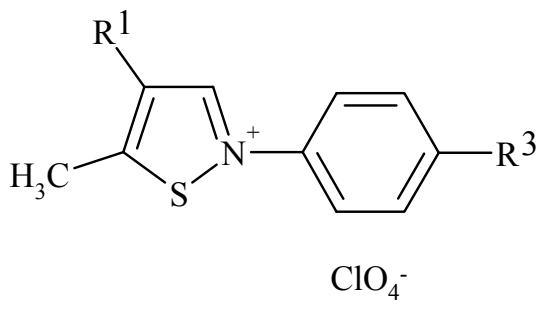

4

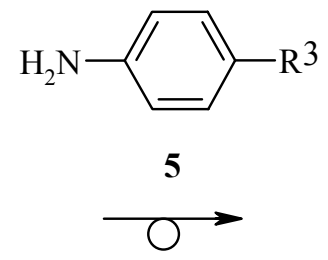

6

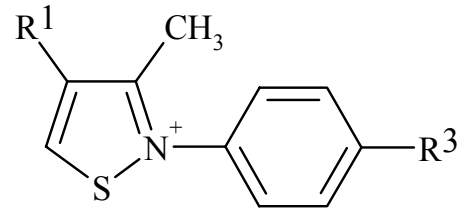

$\mathrm{ClO}_{4}^{-}$

Scheme 2. Ring transformation of isothiazolium salts 4 to 6 .

The oxidation of unsubstituted $\left(\mathrm{R}^{3}=\mathrm{H}\right)$ and donor substituted $\left(\mathrm{R}^{3}=\mathrm{OCH}_{3}, \mathrm{CH}_{3}\right)$ salts $\mathbf{6 a - c}$, f, g with hydrogen peroxide (30\%) in acetic acid at $50^{\circ} \mathrm{C}$ gave after 8 hrs stable 3-hydroperoxysultams 7a-c,f,g in 19-81\% yield (Scheme 3). Surprisingly, the 
3-hydroperoxysultams with acceptor groups $\mathbf{7 d}$, e $\left(\mathrm{R}^{3}=4-\mathrm{Cl}, 4-\mathrm{Br}\right)$ could only be isolated as a mixture with methylenesultams 11d,e (Scheme 3). That gave us reason to investigate the formation of these new compounds.
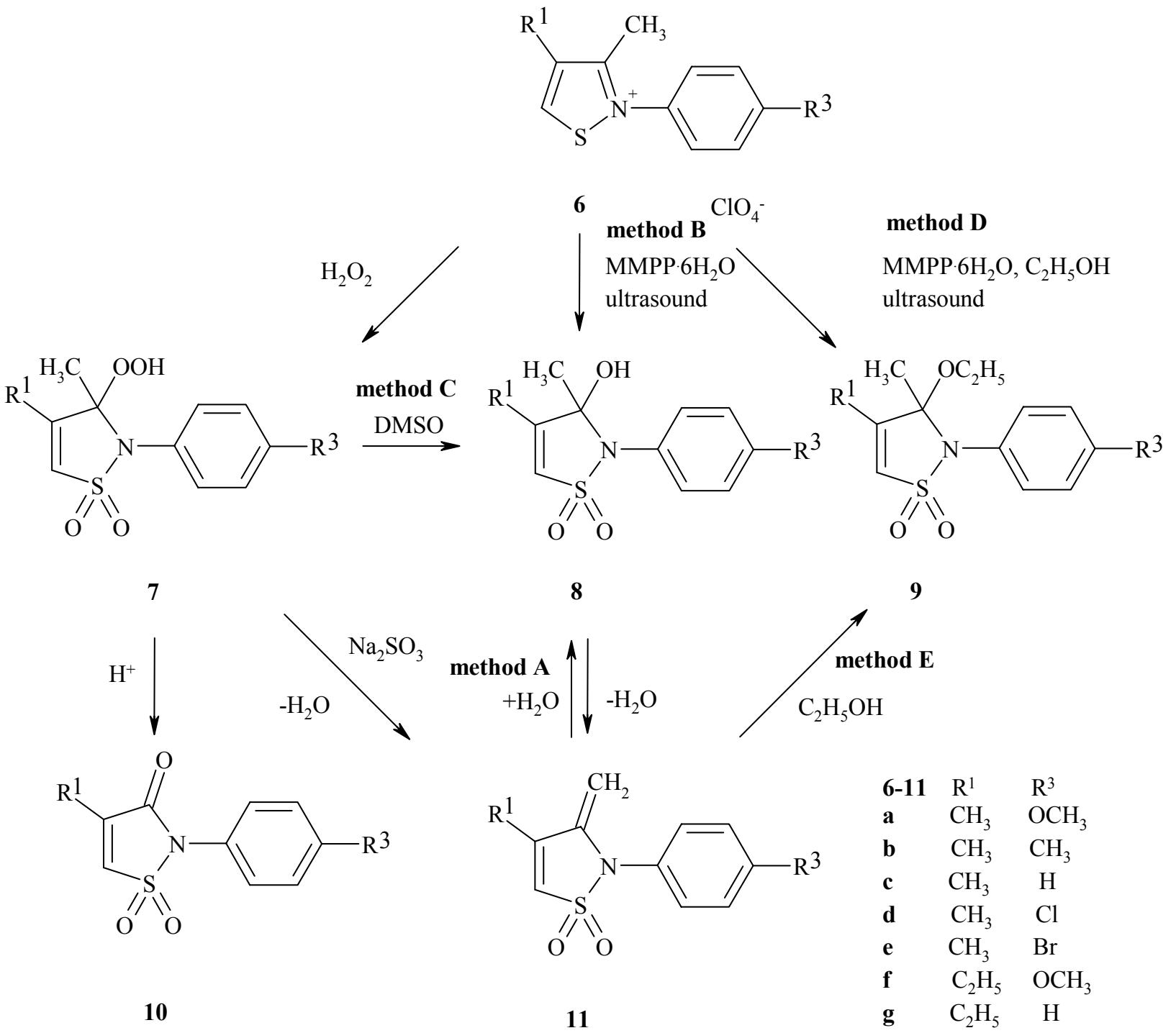

Scheme 3. Products 7-11 of the oxidation of isothazolium salts 6 .

Here we present for the first time the synthesis of the stable, crystalline 2-aryl-3-methylene2,3-dihydroisothiazole1,1-dioxides 11a-c,g formed by reduction of the hydroperoxides 7a-e,g with $\mathrm{Na}_{2} \mathrm{SO}_{3} \cdot 7 \mathrm{H}_{2} \mathrm{O}$ at r.t. for $24 \mathrm{hrs}$. We interpret the formation of 3-methylenesultams 11 as a two-step process. The reduction of 3-hydroperoxysultams 7 takes place in situ to give nonisolable 3-hydroxy-3-methylsultams 8 under these reaction conditions, which directly react by elimination of water to $\mathbf{1 1}$ a-e, $\mathbf{g}$ with a yield range of 10-86\% (poor to good yields). Acceptor substituted 3-methylenesultams $11 \mathbf{d}, \mathbf{e}\left(\mathrm{R}^{3}=4-\mathrm{Cl}, 4-\mathrm{Br}\right)$ could only be isolated in a mixture with 
3-hydroperoxysultams 7d,e. Until now, only one example was known of a dihydro-2-methyl-3methylenthieno[2,3- $d$ ] isothiazole 1,1-dioxide, prepared from 3-thioxothieno[2,3- $d]$ isothiazole 1,1-dioxide and diazomethane. ${ }^{11}$

Exomethylenesultams 11 are favourable building blocks for the oxyfunctionalization in the 3-position. For the first time we obtained 3-alkyl substituted 3-hydroxysultams 8a-c,f,g as stable colourless crystals by the addition of water to a solution of $\mathbf{1 1}$ resulting in good to very good yields in the range of $40-99 \%$ (method A). We also were able to synthesize $\mathbf{8 a}, \mathbf{b}$ in the presence of DMSO from the 3-hydroperoxides 7a,b (method C). Finally, we investigated the oxidation of the salts 6 with MMPP $6 \mathrm{H}_{2} \mathrm{O}$ in an ultrasonic bath at $50^{\circ} \mathrm{C}(3 \mathrm{hrs})$ as previously described for monocyclic 4,5-dimethylisothiazolium salts, ${ }^{9}$ and obtained 3-hydroxy-3-methylsultam 8a with $57 \%$ yield (method $\mathrm{B}$ ). In contrast to the oxidation-reduction method with $\mathrm{Na}_{2} \mathrm{SO}_{3}$ followed by elimination of water to produce 11a-e,g (method A), the method B starting from salts 6 was very convenient (Table 1). In the case of the 3-hydroxysultam 8c we observed the formation of 3methylenesultam 11c from 8c by NMR-detection in DMSO- $\mathrm{d}_{6}$ at $120^{\circ} \mathrm{C}$ within $2 \mathrm{hrs}$.

Table 1. Yield (\%) of products 7-11

\begin{tabular}{cccccccc}
\hline & $\mathbf{R}^{\mathbf{1}}$ & $\mathbf{R}^{\mathbf{3}}$ & $\mathbf{7}^{\mathrm{c}}$ & $\mathbf{8}^{\mathrm{c}}$ & $\mathbf{9}^{\mathrm{h}}$ & $\mathbf{1 0}$ & $\mathbf{1 1}$ \\
\hline $\mathbf{b}$ & $\mathrm{Me}$ & $4-\mathrm{MeO}$ & $81^{10}$ & $70^{\mathrm{d}}$ & $60^{\mathrm{i}}$ & $36^{10}$ & 44 \\
$\mathbf{b}$ & $\mathrm{Me}$ & $4-\mathrm{Me}$ & 52 & $63^{\mathrm{e}}$ & & 33 & 63 \\
$\mathbf{c}$ & $\mathrm{Me}$ & $\mathrm{H}$ & $67^{10}$ & 81 & 66 & $47^{10}$ & 55 \\
$\mathbf{d}$ & $\mathrm{Me}$ & $4-\mathrm{Cl}$ & $27^{\mathrm{a}}$ & $99^{\mathrm{f}}$ & & 10 & $10^{\mathrm{j}}$ \\
$\mathbf{e}$ & $\mathrm{Me}$ & $4-\mathrm{Br}$ & $6^{\mathrm{b}}$ & & & 16 & $12^{\mathrm{k}}$ \\
$\mathbf{f}$ & $\mathrm{Et}$ & $4-\mathrm{MeO}$ & 33 & $99^{\mathrm{g}}$ & & 9 & \\
$\mathbf{g}$ & $\mathrm{Et}$ & $\mathrm{H}$ & 19 & 40 & & 21 & 86 \\
\hline
\end{tabular}

${ }^{a} 3: 1$ mixture with 11 d. ${ }^{b} 1: 2$ mixture with 11e. ${ }^{c}$ method A. ${ }^{d} 57 \%$ by using method B / 48\% by using method C. ${ }^{\mathrm{e}} 53 \%$ by using method C. ${ }^{\mathrm{f}}$ solution of $7 \mathbf{d}$ in DMSO- $d_{6} .{ }^{\mathrm{g}}$ solution of $7 \mathbf{f}$ in DMSO- $d_{6} .{ }^{\mathrm{h}}$ method E. ${ }^{\mathrm{i}} 23 \%$ by using method D. ${ }^{\mathrm{j}} 1: 3$ mixture with 7 d. ${ }^{\mathrm{k}} 2: 1$ mixture with $7 \mathrm{e}$.

Furthermore, when the salt $\mathbf{6 a}$ is reacted with $\mathrm{MMPP} 6 \mathrm{H}_{2} \mathrm{O}$ in ethanol in an ultrasonic bath (method D), the 3-ethoxysultam 9a was synthesized after $3 \mathrm{hrs}$ resulting in a yield of $23 \%$. In contrast, refluxing of the exomethylenesultams 11a,c in ethanol produced 3-ethoxysultams 9a,c resulting in good yields in a range of $60-63 \%($ method $\mathrm{E})$.

Finally the oxidation of the salts $\mathbf{6 b}, \mathbf{d - g}$ with hydrogen peroxide at $70{ }^{\circ} \mathrm{C}$ afforded the 3 oxosultams 10b,d-g as we have already described for $\mathbf{6 a , c .}{ }^{10}$ The mechanism of this reaction is explained by formation of 3-hydroperoxysultams 7, which are not isolated, but give stable 3oxosultams 10 by elimination of water and methanol. The structure of these compounds was confirmed by X-ray structure analysis for derivative 10a in a former paper. ${ }^{10}$ 


\section{Conclusions}

In summary, the oxidation of isothiazolium salts 6 with $\mathrm{H}_{2} \mathrm{O}_{2}$ gives new 3-hydroperoxysultams 7 and 3-oxosultams 10. A new efficient method is introduced for the synthesis of 3-hydroxy- and 3-alkoxysultams 8 and 9 in a one-step reaction of isothiazolium salts 6 with MMPP $6 \mathrm{H}_{2} \mathrm{O}$, and also in a multi-step reaction via novel 3-methylene-functionalized sultams 11.

\section{Experimental Section}

General. Melting points were measured on a Boetius micro-melting-point apparatus and are corrected. ${ }^{1} \mathrm{H}$ - and ${ }^{13} \mathrm{C}-\mathrm{NMR}$ spectra were recorded on a Varian Gemini-200MHz spectrometer using deuterochloroform or DMSO- $\mathrm{d}_{6}$ as solvent and with TMS as internal standard; $\delta$ values are recorded in ppm. IR spectra were recorded on a Genesis FTIR Unicam Analytical System (ATI Mattson) as KBr-pellets; $v_{\max }$ are in $\mathrm{cm}^{-1}$. Mass spectra were performed on a Quadrupole-MS VG 12-250 operating at an ionization potential of $70 \mathrm{eV}$, and elemental analyses were performed on a Heraeus CHNO Rapid Analyzer.

General procedure for the preparation of 4,5-dialkyl-2-aryl-isothiazolium perchlorates (4) and 3,4-dialkyl-2-aryl-isothiazolium perchlorates (6)

The isothiazolium salts $\mathbf{4}$ and $\mathbf{6}$ were prepared according to the procedure described. ${ }^{10,12,13}$

General procedure for the preparation of 2-aryl-3-hydroperoxy-2,3-dihydroisothiazole 1,1dioxides (7)

The new 3-hydroperoxysultams $\mathbf{7 b}, \mathbf{d}-\mathbf{g}$ were prepared according to the procedure described. ${ }^{10}$

3-Hydroperoxy-3,4-dimethyl-2-(4-methylphenyl)-2,3-dihydroisothiazole 1,1-dioxide (7b). Yield: $52 \%, 0.14 \mathrm{~g}$. colourless crystals. m.p. $87-91^{\circ} \mathrm{C}$. IR $\left(\mathrm{KBr}\right.$-pellets, $\left.v_{\max }, \mathrm{cm}^{-1}\right): 1168\left(\mathrm{SO}_{2}\right)$, $1276\left(\mathrm{SO}_{2}\right) .{ }^{1} \mathrm{H}-\mathrm{NMR}\left(200 \mathrm{MHz}, \mathrm{CDCl}_{3}\right): \delta=1.34\left(\mathrm{~s}, 3 \mathrm{H}, \mathrm{CH}_{3}\right) ; 2.08\left(\mathrm{~s}, 3 \mathrm{H}, \mathrm{CH}_{3}\right) ; 2.38(\mathrm{~s}, 3 \mathrm{H}$, p- $\left.\mathrm{CH}_{3}\right) ; 6.60(\mathrm{~s}(\mathrm{br}), 1 \mathrm{H},=\mathrm{CH}-5) ; 7.23\left(\mathrm{~d}, J_{\mathrm{AB}}=8.0 \mathrm{~Hz}, 2 \mathrm{H}\right.$, arom. $\left.\left.\mathrm{H}\right)\right) ; 7.39\left(\mathrm{~d}, J_{\mathrm{AB}}=8.0 \mathrm{~Hz}, 2 \mathrm{H}\right.$, arom. H). ${ }^{13} \mathrm{C}-\mathrm{NMR}\left(50 \mathrm{MHz}, \mathrm{CDCl}_{3}\right): \delta=13.7\left(\mathrm{CH}_{3}\right) ; 19.8\left(\mathrm{CH}_{3}\right) ; 21.9\left(p-\mathrm{CH}_{3}\right) ; 98.3(\mathrm{C}-3)$; 124.7 (CH-5); $128.4(i-\mathrm{C}) ; 130.9(o-\mathrm{CH}) ; 131.3(m-\mathrm{CH}) ; 139.9$ (p-C); 149.6 (C-4). MS (EI $70 \mathrm{eV}): m / z(\%)=251.0\left(\left[\mathrm{M}^{+}-\mathrm{H}_{2} \mathrm{O}\right]^{+}\right)$. Anal. Calcd for $\mathrm{C}_{11} \mathrm{H}_{13} \mathrm{NO}_{4} \mathrm{~S}(269.3) \mathrm{C}: 53.52, \mathrm{H} 5.61, \mathrm{~N}$ 5.20; O 23.76; found C 53.49, H 5.43, N 5.34, O 23.60\%.

2-(4-Chlorophenyl)-3-hydroperoxy-3,4-dimethyl-2,3-dihydroisothiazole 1,1-dioxide (7d). Yield: $27 \%, 0.07 \mathrm{~g}(3: 1$ mixture with $11 \mathrm{~d})$. colourless crystals. m.p. $94-98^{\circ} \mathrm{C}$. IR (KBr-pellets, $\left.v_{\max }, \mathrm{cm}^{-1}\right): 1171\left(\mathrm{SO}_{2}\right), 1276\left(\mathrm{SO}_{2}\right) .{ }^{1} \mathrm{H}-\mathrm{NMR}\left(200 \mathrm{MHz}, \mathrm{CDCl}_{3}\right): \delta=1.32\left(\mathrm{~s}, 3 \mathrm{H}, \mathrm{CH}_{3}\right) ; 2.06(\mathrm{~s}$, $\left.3 \mathrm{H}, \mathrm{CH}_{3}\right) ; 6.60(\mathrm{~s}(\mathrm{br}), 1 \mathrm{H},=\mathrm{CH}-5) ; 7.38\left(\mathrm{~d}, J_{\mathrm{AB}}=9.20 \mathrm{~Hz}, 2 \mathrm{H}\right.$, arom. $\left.\mathrm{H}\right) ; 7.48\left(\mathrm{~d}, J_{\mathrm{AB}}=9.21 \mathrm{~Hz}\right.$, $2 \mathrm{H}$, arom. H). ${ }^{13} \mathrm{C}-\mathrm{NMR}\left(50 \mathrm{MHz}, \mathrm{CDCl}_{3}\right): \delta=13.3\left(\mathrm{CH}_{3}\right) ; 19.5\left(\mathrm{CH}_{3}\right) ; 98.1(\mathrm{C}-3) ; 125.4(\mathrm{CH}-$ 
5); $130.2(o-\mathrm{CH}) ; 131.5(i-\mathrm{C}) ; 132.2(m-\mathrm{CH}) ; 135.6(p-\mathrm{C}) ; 149.7(\mathrm{C}-4)$. MS (EI 70eV): $\mathrm{m} / z(\%)=$ $271.0\left(\left[\mathrm{M}^{+}-\mathrm{H}_{2} \mathrm{O}\right]^{+}\right) \cdot \mathrm{C}_{11} \mathrm{H}_{12} \mathrm{ClNO}_{4} \mathrm{~S}(289.7)$.

2-(4-Bromophenyl)-3-hydroperoxy-3,4-dimethyl-2,3-dihydroisothiazole 1,1-dioxide (7e). Yield: $6 \%$, 0.02g (2:1 mixture with 11e). colourless crystals. m.p. 102-105 ${ }^{\circ} \mathrm{C}$. IR (KBr-pellets, $\left.v_{\max }, \mathrm{cm}^{-1}\right): 1171\left(\mathrm{SO}_{2}\right), 1277\left(\mathrm{SO}_{2}\right) .{ }^{1} \mathrm{H}-\mathrm{NMR}\left(200 \mathrm{MHz}, \mathrm{CDCl}_{3}\right): \delta=1.37\left(\mathrm{~s}, 3 \mathrm{H}, \mathrm{CH}_{3}\right) ; 2.10(\mathrm{~s}$, $\left.3 \mathrm{H}, \mathrm{CH}_{3}\right) ; 6.63(\mathrm{~s}(\mathrm{br}), 1 \mathrm{H},=\mathrm{CH}-5) ; 7.44\left(\mathrm{~d}, J_{\mathrm{AB}}=8.6 \mathrm{~Hz}, 2 \mathrm{H}\right.$, arom. H $) ; 7.54\left(\mathrm{~d}, J_{\mathrm{AB}}=8.6 \mathrm{~Hz}\right.$, $2 \mathrm{H}$, arom. H) MS (EI 70eV): $m / z(\%)=316.0\left(\left[\mathrm{M}^{+}-\mathrm{H}_{2} \mathrm{O}\right]^{+}\right), \mathrm{C}_{11} \mathrm{H}_{12} \mathrm{BrNO}_{4} \mathrm{~S}(334.2)$.

4-Ethyl-3-hydroperoxy-2-(4-methoxyphenyl)-3-methyl-2,3-dihydroisothiazole 1,1-dioxide (7f). Yield: $33 \%, 0.09$ g. colourless crystals. m.p. $115-118^{\circ} \mathrm{C}$. IR (KBr-pellets, $\left.v_{\max }, \mathrm{cm}^{-1}\right): 1165$ $\left(\mathrm{SO}_{2}\right), 1249\left(\mathrm{OCH}_{3}\right), 1284\left(\mathrm{SO}_{2}\right) .{ }^{1} \mathrm{H}-\mathrm{NMR}\left(200 \mathrm{MHz}, \mathrm{CDCl}_{3}\right): \delta=1.27\left(\mathrm{t},{ }^{3} \mathrm{~J}=7.2 \mathrm{~Hz}, 3 \mathrm{H}, \mathrm{CH}_{3}\right)$; $1.34\left(\mathrm{~s}, 3 \mathrm{H}, \mathrm{CH}_{3}\right) ; 2.32-2.36\left(\mathrm{~m}, 1 \mathrm{H}, \mathrm{CH}_{2}^{\mathrm{a}}\right) ; 2.46-2.50\left(\mathrm{~m}, 1 \mathrm{H}, \mathrm{CH}_{2}{ }^{\mathrm{b}}\right) ; 3.83\left(\mathrm{~s}, 3 \mathrm{H}, \mathrm{OCH}_{3}\right) ; 6.61$ (s(br), 1H, =CH-5); $6.96\left(\mathrm{~d}, J_{\mathrm{AB}}=8.8 \mathrm{~Hz}, 2 \mathrm{H}\right.$, arom. H ); $7.41(\mathrm{~d}, J=8.8 \mathrm{~Hz}, 2 \mathrm{H}$, arom. H); 8.39 $(\mathrm{s}, 1 \mathrm{H}, \mathrm{OOH}) ;{ }^{13} \mathrm{C}-\mathrm{NMR}\left(50 \mathrm{MHz}, \mathrm{CDCl}_{3}\right): \delta=11.3\left(\mathrm{CH}_{3}\right) ; 20.0\left(\mathrm{CH}_{3}\right) ; 20.6\left(\mathrm{CH}_{2}\right) ; 98.4(\mathrm{C}-3)$; 115.4 (m-CH); 122.9 ( $i$-C); $123.4(\mathrm{CH}-5) ; 133.5(o-\mathrm{CH}) ; 155.6$ (C-4); 160.9 (p-C). MS (EI $70 \mathrm{eV}): m / z(\%)=265.0\left(\left[\mathrm{M}^{+}-\mathrm{H}_{2} \mathrm{O},-\mathrm{CH}_{3}\right]^{+}\right)$. Anal. Calcd for $\mathrm{C}_{13} \mathrm{H}_{17} \mathrm{NO}_{5} \mathrm{~S}$ (299.3) C: 52.16, $\mathrm{H}$ 5.72, N 4.68; O 26.72; found C 52.46, H 5.63, N 4.81, O 26.76\%.

4-Ethyl-3-hydroperoxy-3-methyl-2-phenyl-2,3-dihydroisothiazole 1,1-dioxide (7g). Yield: $19 \%, 0.05$ g. colourless crystals. m.p. $109-111^{\circ} \mathrm{C}$. IR (KBr-pellets, $\left.v_{\max }, \mathrm{cm}^{-1}\right): 1164\left(\mathrm{SO}_{2}\right), 1287$ $\left(\mathrm{SO}_{2}\right)$. ${ }^{1} \mathrm{H}-\mathrm{NMR}\left(200 \mathrm{MHz}, \mathrm{CDCl}_{3}\right): \delta=1.24\left(\mathrm{t},{ }^{3} \mathrm{~J}=7.3 \mathrm{~Hz}, 3 \mathrm{H}, \mathrm{CH}_{3}\right) ; 1.35\left(\mathrm{~s}, 3 \mathrm{H}, \mathrm{CH}_{3}\right) ; 2.26-$ $2.36\left(\mathrm{~m}, 1 \mathrm{H}, \mathrm{CH}_{2}{ }^{\mathrm{a}}\right) ; 2.43-2.57\left(\mathrm{~m}, 1 \mathrm{H}, \mathrm{CH}_{2}{ }^{\mathrm{b}}\right) ; 6.57(\mathrm{~s}(\mathrm{br}), 1 \mathrm{H},=\mathrm{CH}-5) ; 7.40-7.45(\mathrm{~m}, 3 \mathrm{H}$, arom. $\mathrm{H}) ; 7.52-7.55\left(\mathrm{~m}, 2 \mathrm{H}\right.$, arom. H). ${ }^{13} \mathrm{C}-\mathrm{NMR}\left(50 \mathrm{MHz}, \mathrm{CDCl}_{3}\right): \delta=12.3\left(\mathrm{CH}_{3}\right) ; 20.0\left(\mathrm{CH}_{3}\right) ; 20.9$ $\left(\mathrm{CH}_{2}\right) ; 98.6(\mathrm{C}-3) ; 123.5(\mathrm{CH}-5) ; 129.6(p-\mathrm{CH}) ; 130.2(o-\mathrm{CH}) ; 131.1(m-\mathrm{CH}) ; 131.4(i-\mathrm{C}) ; 155.7$ $(\mathrm{C}-4)$. MS (EI 70eV): $m / z(\%)=269.0\left(\mathrm{M}^{+}\right)$. Anal. Calcd for $\mathrm{C}_{12} \mathrm{H}_{15} \mathrm{NO}_{4} \mathrm{~S}$ (269.3) C: 53.52, $\mathrm{H}$ 5.61, N 5.20; O 23.76; found C 52.83, H 5.47, N 5.29, O 23.60\%.

General procedure for the preparation of 2-aryl-3-hydroxy-2,3-dihydroisothiazole 1,1dioxides (8)

Method A. 0.5 mmol 3-methylene-2,3-dihydroisothiazole 1,1-dioxide 11 is dissolved in $10 \mathrm{~mL}$ of a 1:1 mixture of ethanol and distilled water and refluxed for 5-10 minutes. By slow removal of a little solvent, crystals of $\mathbf{8}$ are obtained, filtered off and dried.

Method B. $0.25 \mathrm{mmol}$ isothiazolium salt 6 is dissolved in $4 \mathrm{~mL}$ of a 3:1 mixture of acetonitrile and water. Then $1.5 \mathrm{mmol} \mathrm{MMPP} 6 \mathrm{H}_{2} \mathrm{O}$ is added and the mixture is stirred for $3 \mathrm{hrs}$ at $50^{\circ} \mathrm{C}$ in an ultrasonic bath. To the mixture is given sat. $\mathrm{NaHCO}_{3}$ solution and extracted with diethylether (3x). The combined organic layers are dried over anhydrous $\mathrm{MgSO}_{4}$. The solvent is evaporated and $\mathbf{8}$ is purified by recrystallization from ethanol.

Method C. $0.5 \mathrm{mmol}$ 3-hydroperoxysultam 7 are dissolved in $2 \mathrm{~mL}$ DMSO. After $2 \mathrm{hrs}$ standing at room temperature the solution of $\mathbf{8}$ was lyophilized and the precipitated 3-hydroxysultams $\mathbf{8}$ were filtered off and are purified by recrystallization from acetone.

3-Hydroxy-2-(4-methoxyphenyl)-3,4-dimethyl-2,3-dihydroisothiazole 1,1-dioxide (8a). Yield: 70\%, $0.09 \mathrm{~g}(\operatorname{method} \mathrm{A}) / 57 \%, 0.04 \mathrm{~g}(\operatorname{method} \mathrm{B}) / 48 \%, 0.06 \mathrm{~g}(\operatorname{method} \mathrm{C})$. Colourless 
needles. m.p. $150-153^{\circ} \mathrm{C}$. IR (KBr-pellets, $\left.v_{\max }, \mathrm{cm}^{-1}\right): 1140\left(\mathrm{SO}_{2}\right), 1221\left(\mathrm{OCH}_{3}\right), 1278\left(\mathrm{SO}_{2}\right)$. ${ }^{1} \mathrm{H}-\mathrm{NMR}\left(200 \mathrm{MHz}, \mathrm{CDCl}_{3}\right): \delta=1.42\left(\mathrm{~s}, 3 \mathrm{H}, \mathrm{CH}_{3}\right) ; 2.11\left(\mathrm{~s}, 3 \mathrm{H}, \mathrm{CH}_{3}\right) ; 3.32(\mathrm{~s}, 1 \mathrm{H}, \mathrm{OH}) ; 3.83(\mathrm{~s}$, $\left.3 \mathrm{H}, \mathrm{OCH}_{3}\right) ; 6.53(\mathrm{~s}(\mathrm{br}), 1 \mathrm{H},=\mathrm{CH}-5) ; 6.69\left(\mathrm{~d}, J_{\mathrm{AB}}=8.9 \mathrm{~Hz}, 2 \mathrm{H}, \operatorname{arom} . \mathrm{H}\right) ; 7.40\left(\mathrm{~d}, J_{\mathrm{AB}}=8.9 \mathrm{~Hz}\right.$, 2H, arom. H). ${ }^{13} \mathrm{C}-\mathrm{NMR}\left(50 \mathrm{MHz}, \mathrm{CDCl}_{3}\right): \delta=13.7\left(\mathrm{CH}_{3}\right) ; 23.5\left(\mathrm{CH}_{3}\right) ; 56.1\left(\mathrm{OCH}_{3}\right) ; 90.1(\mathrm{C}-3)$; $115.3(m-\mathrm{CH}) ; 122.8(\mathrm{CH}-5) ; 123.2(i-\mathrm{C}) ; 134.1(o-\mathrm{CH}) ; 161.0(\mathrm{C}-4) ; 164.1(p-\mathrm{C})$. MS (EI $70 \mathrm{eV}): m / z(\%)=269.0\left(\mathrm{M}^{+}\right)$. Anal. Calcd for $\mathrm{C}_{12} \mathrm{H}_{15} \mathrm{NO}_{4} \mathrm{~S}$ (269.3) C: 53.52, H 5.61, N 5.20; O 23.76; found C 53.47, H 5.87, N 5.52, O 23.70\%.

3-Hydroxy-3,4-dimethyl-2-(4-methylphenyl)-2,3-dihydroisothiazole 1,1-dioxide (8b). Yield: $63 \%, 0.08 \mathrm{~g}(\operatorname{method} \mathrm{A}) / 53 \%, 0.07 \mathrm{~g}(\operatorname{method} \mathrm{C})$. colourless needles. m.p. $122-124^{\circ} \mathrm{C}$. IR $(\mathrm{KBr}-$ pellets, $\left.v_{\max }, \mathrm{cm}^{-1}\right): 1145\left(\mathrm{SO}_{2}\right), 1274\left(\mathrm{SO}_{2}\right) .{ }^{1} \mathrm{H}-\mathrm{NMR}\left(200 \mathrm{MHz}, \mathrm{CDCl}_{3}\right): \delta=1.40\left(\mathrm{~s}, 3 \mathrm{H}, \mathrm{CH}_{3}\right)$; $2.07\left(\mathrm{~s}, 3 \mathrm{H}, \mathrm{CH}_{3}\right) ; 2.39$ (s, $\left.3 \mathrm{H}, p-\mathrm{CH}_{3}\right) ; 3.64(\mathrm{~s}, 1 \mathrm{H}, \mathrm{OH}) ; 6.51(\mathrm{~s}(\mathrm{br}), 1 \mathrm{H},=\mathrm{CH}-5) ; 7.18\left(\mathrm{~d}, J_{\mathrm{AB}}=\right.$ $8.2 \mathrm{~Hz}, 2 \mathrm{H}$, arom. H); 7.37 ( d, $J_{\mathrm{AB}}=8.2 \mathrm{~Hz}$., $2 \mathrm{H}$, arom. H). ${ }^{13} \mathrm{C}-\mathrm{NMR}\left(50 \mathrm{MHz}, \mathrm{CDCl}_{3}\right): \delta=$ $13.7\left(\mathrm{CH}_{3}\right) ; 21.6\left(p-\mathrm{CH}_{3}\right) ; 23.5\left(\mathrm{CH}_{3}\right) ; 90.3(\mathrm{C}-3) ; 115.3(m-\mathrm{CH}) ; 122.6(\mathrm{CH}-5) ; 128.5(i-\mathrm{C})$; $131.8(o-\mathrm{CH}) ; 139.6(p-\mathrm{C}) ; 152.6(\mathrm{C}-4)$. MS (EI 70eV): $\mathrm{m} / \mathrm{z}(\%)=253.0\left(\mathrm{M}^{+}\right)$. Anal. Calcd for $\mathrm{C}_{12} \mathrm{H}_{15} \mathrm{NO}_{3} \mathrm{~S}$ (253.3) C: 56.90, H 5.97, N 5.53; O 18.95; found C 56.59, H 5.72, N 5.43, O $19.20 \%$.

3-Hydroxy-3,4-dimethyl-2-phenyl-2,3-dihydroisothiazole 1,1-dioxide (8c). Yield: 81\%, 0.09g (method A). colourless needles. mp 128-131 ${ }^{\circ} \mathrm{C}$. IR (KBr-pellets, $\left.v_{\max }, \mathrm{cm}^{-1}\right): 1139\left(\mathrm{SO}_{2}\right), 1284$ $\left(\mathrm{SO}_{2}\right) .{ }^{1} \mathrm{H}-\mathrm{NMR}\left(200 \mathrm{MHz}, \mathrm{CDCl}_{3}\right): \delta=1.44\left(\mathrm{~s}, 3 \mathrm{H}, \mathrm{CH}_{3}\right) ; 2.12\left(\mathrm{~s}, 3 \mathrm{H}, \mathrm{CH}_{3}\right) ; 3.23(\mathrm{~s}, 1 \mathrm{H}, \mathrm{OH})$; $6.53(\mathrm{~s}(\mathrm{br}), 1 \mathrm{H},=\mathrm{CH}-5)$; 7.44-7.47 (m, 3H, arom. $\mathrm{H}) ; 7.51-7.53(\mathrm{~m}, 2 \mathrm{H}$ arom. $\mathrm{H}) .{ }^{13} \mathrm{C}-\mathrm{NMR}$ $\left(50 \mathrm{MHz}, \mathrm{CDCl}_{3}\right): \delta=13.6\left(\mathrm{CH}_{3}\right) ; 23.5\left(\mathrm{CH}_{3}\right) ; 90.5(\mathrm{C}-3) ; 123.0(\mathrm{CH}-5) ; 129.7(p-\mathrm{C}) ; 130.1(o-$ $\mathrm{CH}) ; 131.6(i-\mathrm{C}) ; 132.0(\mathrm{~m}-\mathrm{CH}) ; 152.5(\mathrm{C}-4)$. MS (EI 70eV): $\mathrm{m} / z(\%)=239.0\left(\mathrm{M}^{+}\right)$. Anal. Calcd for $\mathrm{C}_{11} \mathrm{H}_{13} \mathrm{NO}_{3} \mathrm{~S}$ (239.3) C: 55.21, H 5.48, N 5.85; O 20.06; found C 54.77, H 5.59, N 5.82, O $20.80 \%$.

2-(4-Chlorphenyl)-3-hydroxy-3,4-dimethyl-2,3-dihydroisothiazole 1,1-dioxide (8d). Yield: $99 \%$ (solution from 7d in DMSO- $\left.d_{6}\right) .{ }^{1} \mathrm{H}-\mathrm{NMR}\left(200 \mathrm{MHz}, \mathrm{DMSO}-d_{6}\right): \delta=1.32\left(\mathrm{~s}, 3 \mathrm{H}, \mathrm{CH}_{3}\right)$; $2.04\left(\mathrm{~s}, 3 \mathrm{H}, \mathrm{CH}_{3}\right) ; 7.13(\mathrm{~s}(\mathrm{br}), 1 \mathrm{H},=\mathrm{CH}-5) ; 7.50\left(\mathrm{~d}, J_{\mathrm{AB}}=9.0 \mathrm{~Hz}, 2 \mathrm{H}\right.$, arom.H); $7.57\left(\mathrm{~d}, J_{\mathrm{AB}}=\right.$ $9.0 \mathrm{~Hz}, 2 \mathrm{H}$, arom. H). ${ }^{13} \mathrm{C}-\mathrm{NMR}\left(50 \mathrm{MHz}, \mathrm{DMSO}-d_{6}\right): \delta=12.6\left(\mathrm{CH}_{3}\right) ; 23.8\left(\mathrm{CH}_{3}\right) ; 90.3(\mathrm{C}-3)$; $122.0 \quad(\mathrm{CH}-5) ; 129.4 \quad(o-\mathrm{CH}) ; 131.8 \quad(m-\mathrm{CH}) ; 132.8$ (i-C); $141.3 \quad(p-\mathrm{C}) ; 152.8 \quad(\mathrm{C}-4)$. $\mathrm{C}_{11} \mathrm{H}_{12} \mathrm{ClNO}_{3} \mathrm{~S}(273.7)$

4-Ethyl-3-hydroxy-2-(4-methoxylphenyl)-3-methyl-2,3-dihydroisothiazole 1,1-dioxide (8f). Yield: 99\% (solution from 7d in DMSO- $\left.d_{6}\right)$. ${ }^{1} \mathrm{H}-\mathrm{NMR}\left(200 \mathrm{MHz}, \mathrm{DMSO}-d_{6}\right): \delta=1.15\left(\mathrm{t},{ }^{3} J=\right.$ $\left.7.3 \mathrm{~Hz}, 3 \mathrm{H}, \mathrm{CH}_{3}\right) ; 1.25$ (s, $\left.3 \mathrm{H}, \mathrm{CH}_{3}\right) ; 2.39\left(\mathrm{~m}, 2 \mathrm{H}, \mathrm{CH}_{2}\right) ; 3.78\left(\mathrm{~s}, 3 \mathrm{H}, \mathrm{OCH}_{3}\right) ; 7.04(\mathrm{~s}(\mathrm{br}), 1 \mathrm{H}$, CH-5); $7.02\left(\mathrm{~d}, J_{\mathrm{AB}}=8.9 \mathrm{~Hz}, 2 \mathrm{H}\right.$, arom. H ), $7.31\left(\mathrm{~d}, J_{\mathrm{AB}}=8.9 \mathrm{~Hz}, 2 \mathrm{H}\right.$, arom.H). ${ }^{13} \mathrm{C}-\mathrm{NMR}$ (50MHz, DMSO-d $\left.d_{6}\right): \delta=10.8\left(\mathrm{CH}_{3}\right) ; 19.4\left(\mathrm{CH}_{2}\right) ; 24.0\left(\mathrm{CH}_{3}\right) ; 55.3\left(\mathrm{OCH}_{3}\right) ; 89.4(\mathrm{C}-3) ; 114.2$ $(m-\mathrm{CH}) ; 120.5(\mathrm{CH}-5) ; 123.4(i-\mathrm{C}) ; 133.3(o-\mathrm{CH}) ; 158.3(p-\mathrm{C}) ; 159.4(\mathrm{C}-4) . \mathrm{C}_{13} \mathrm{H}_{17} \mathrm{NO}_{4} \mathrm{~S}$ (283.3)

4-Ethyl-3-hydroxy-3-methyl-2-phenyl-2,3-dihydroisothiazole 1,1-dioxide (8g). Yield: 40\%, $0.05 \mathrm{~g}(\operatorname{method} \mathrm{A})$. colourless needles. m.p. $93-98^{\circ} \mathrm{C}$. IR (KBr-pellets, $\left.v_{\max }, \mathrm{cm}^{-1}\right): 1128\left(\mathrm{SO}_{2}\right)$, $1265\left(\mathrm{SO}_{2}\right) .{ }^{1} \mathrm{H}-\mathrm{NMR}\left(200 \mathrm{MHz}, \mathrm{CDCl}_{3}\right): \delta=1.26\left(\mathrm{t},{ }^{3} J=7.3 \mathrm{~Hz}, 3 \mathrm{H}, \mathrm{CH}_{3}\right) ; 1.43\left(\mathrm{~s}, 3 \mathrm{H}, \mathrm{CH}_{3}\right)$; 
$2.30\left(\mathrm{~m}, 1 \mathrm{H}, \mathrm{CH}_{2}\right) ; 2.41\left(\mathrm{~m}, 1 \mathrm{H}, \mathrm{CH}_{2}\right) ; 3.46(\mathrm{~s}, 1 \mathrm{H}, \mathrm{OH}) ; 6.50(\mathrm{~s}(\mathrm{br}), 1 \mathrm{H},=\mathrm{CH}-5) ; 7.43-7.45(\mathrm{~m}$, $3 \mathrm{H}$ arom. $\mathrm{H}) ; 7.47-7.53\left(\mathrm{~m}, 2 \mathrm{H}\right.$ arom. H). ${ }^{13} \mathrm{C}-\mathrm{NMR}\left(50 \mathrm{MHz}, \mathrm{CDCl}_{3}\right): \delta=11.5\left(\mathrm{CH}_{3}\right) ; 20.6$ $\left(\mathrm{CH}_{2}\right) ; 23.7\left(\mathrm{CH}_{3}\right)$; 90.7 (C-3); $121.3(\mathrm{CH}-5) ; 129.6(p-\mathrm{C}) ; 130.0(o-\mathrm{CH}) ; 131.6(i-\mathrm{C}) ; 132.0(\mathrm{~m}-$ $\mathrm{CH}) ; 158.8(\mathrm{C}-4)$. MS (EI 70eV): $\mathrm{m} / \mathrm{z}(\%)=253.0\left(\mathrm{M}^{+}\right)$. Anal. Calcd for $\mathrm{C}_{12} \mathrm{H}_{15} \mathrm{NO}_{3} \mathrm{~S}(253.3) \mathrm{C}$ : 56.90, H 5.97, N 5.53; O 18.95; found C 57.38, H 6.09, N 5.55, O 19.15\%.

General procedure for the preparation of 2-aryl-3-ethoxy-2,3-dihydroisothiazole 1,1-dioxides (9)

Method D: $0.25 \mathrm{mmol}$ isothiazolium salt 6 is dissolved with $4 \mathrm{~mL}$ ethanol. Then $1.5 \mathrm{mmol}$ MMPP $6 \mathrm{H}_{2} \mathrm{O}$ is added and stirred for $3 \mathrm{hrs}$ at $50^{\circ} \mathrm{C}$ in ultrasonic bath. To the mixture is given sat. $\mathrm{NaHCO}_{3}$-solution and the mixture is extracted with diethylether $(3 \mathrm{x})$. The combined organic layers are dried over $\mathrm{MgSO}_{4}$. The solvent is evaporated and 9 is purified by recrystallization from ethanol.

Method E: 0.5 mmol 3-methylene-2,3-dihydroisothiazole 1,1-dioxide 11 is refluxed with ethanol. After $10 \mathrm{~min}$ a small volume of the solvent is evaporated. Crystals of 9 are obtained, filtered off and dried.

3-Ethoxy-2-(4-methoxyphenyl)-3,4-dimethyl-2,3-dihydroisothiazole 1,1-dioxide (9a). Yield: $23 \%, 0.02 \mathrm{~g}(\operatorname{method} \mathrm{D}) / 60 \% 0.09 \mathrm{~g}(\operatorname{method} \mathrm{E})$. colourless powder. m.p. $108-110^{\circ} \mathrm{C}$. IR $(\mathrm{KBr}-$ pellets, $\left.v_{\max }, \mathrm{cm}^{-1}\right): 1179\left(\mathrm{SO}_{2}\right), 1247\left(\mathrm{OCH}_{3}\right), 1287\left(\mathrm{SO}_{2}\right) .{ }^{1} \mathrm{H}-\mathrm{NMR}\left(200 \mathrm{MHz}, \mathrm{CDCl}_{3}\right): \delta=1.24$ $\left(\mathrm{t},{ }^{3} J=7.2 \mathrm{~Hz}, 3 \mathrm{H}, \mathrm{CH}_{3}\right) ; 1.39\left(\mathrm{~s}, 3 \mathrm{H}, \mathrm{CH}_{3}\right) ; 2.01\left(\mathrm{~d},{ }^{4} \mathrm{~J}=1.8 \mathrm{~Hz}, 3 \mathrm{H}, \mathrm{CH}_{3}\right) ; 3.21\left(\mathrm{dq},{ }^{2} J=12.8\right.$ $\left.\mathrm{Hz},{ }^{3} J=7.2 \mathrm{~Hz}, 1 \mathrm{H}, \mathrm{OCH}_{2}{ }^{\mathrm{a}}\right) ; 3.82\left(\mathrm{dq},{ }^{2} J=12.8 \mathrm{~Hz},{ }^{3} J=7.2 \mathrm{~Hz}, 1 \mathrm{H}, \mathrm{OCH}_{2}{ }^{\mathrm{b}}\right) ; 3.83(\mathrm{~s}, 3 \mathrm{H}$, $\left.\mathrm{OCH}_{3}\right) ; 6.60$ (q, $\left.{ }^{4} \mathrm{~J}=1.8 \mathrm{~Hz}, 1 \mathrm{H},=\mathrm{CH}-5\right) ; 6.95\left(\mathrm{~d},{ }^{3} \mathrm{~J}=9.0 \mathrm{~Hz}, 2 \mathrm{H}\right.$, arom. H $) ; 7.31\left(\mathrm{~d},{ }^{3} J=9.0\right.$ $\mathrm{Hz}, 2 \mathrm{H}$, arom.H). ${ }^{13} \mathrm{C}-\mathrm{NMR}\left(50 \mathrm{MHz}, \mathrm{CDCl}_{3}\right): \delta=13.8\left(\mathrm{CH}_{3}\right) ; 19.1\left(\mathrm{CH}_{3}\right) ; 19.1\left(\mathrm{CH}_{3}\right) ; 23.8$ $\left(\mathrm{CH}_{3}\right) ; 56.1\left(\mathrm{OCH}_{3}\right) ; 59.5\left(\mathrm{CH}_{2}\right) ; 94.4(\mathrm{C}-3) ; 115.3(m-\mathrm{CH}) ; 123.8(i-\mathrm{C}) ; 124.9(\mathrm{CH}-5) ; 132.9$ (o$\mathrm{CH}) ; 150.1(\mathrm{C}-4) ; 160.5(p-\mathrm{C})$. MS (EI 70eV): $\mathrm{m} / z(\%)=297.0\left(\mathrm{M}^{+}\right)$. Anal. Calcd for $\mathrm{C}_{14} \mathrm{H}_{19} \mathrm{NO}_{4} \mathrm{~S}$ (297.3) $\mathrm{C}: 56.55, \mathrm{H}$ 6.44, N 4.71; O 21.52; found $\mathrm{C} 56.80, \mathrm{H} 6.56, \mathrm{~N} 4.72, \mathrm{O}$ $21.70 \%$.

3-Ethoxy-3,4-dimethyl-2-phenyl-2,3-dihydroisothiazole 1,1-dioxide (9c). Yield: 66\%, 0.09g (method E). colourless powder. m.p. 88-90 ${ }^{\circ} \mathrm{C}$. IR (KBr-pellets, $\left.v_{\max }, \mathrm{cm}^{-1}\right): 1179\left(\mathrm{SO}_{2}\right), 1286$ $\left(\mathrm{SO}_{2}\right) .{ }^{1} \mathrm{H}-\mathrm{NMR}\left(200 \mathrm{MHz}, \mathrm{CDCl}_{3}\right): \delta=1.22\left(\mathrm{t},{ }^{3} J=7.3 \mathrm{~Hz}, 3 \mathrm{H}, \mathrm{CH}_{3}\right) ; 1.44\left(\mathrm{~s}, 3 \mathrm{H}, \mathrm{CH}_{3}\right) ; 2.01$ $\left(\mathrm{d},{ }^{4} \mathrm{~J}=1.7 \mathrm{~Hz}, 3 \mathrm{H}, \mathrm{CH}_{3}\right) ; 3.25\left(\mathrm{dq},{ }^{2} J=13.0 \mathrm{~Hz},{ }^{3} \mathrm{~J}=7.3 \mathrm{~Hz}, 1 \mathrm{H}, \mathrm{OCH}_{2}{ }^{\mathrm{a}}\right) ; 3.75\left(\mathrm{dq},{ }^{2} J=13.0 \mathrm{~Hz}\right.$, $\left.{ }^{3} J=7.3 \mathrm{~Hz}, 1 \mathrm{H}, \mathrm{OCH}_{2 \mathrm{~b}}\right) ; 6.60$ (q, $\left.{ }^{4} J=1.7 \mathrm{~Hz}, 1 \mathrm{H},=\mathrm{CH}-5\right) ; 7.38-7.49$ (m, 5H arom. H). ${ }^{13} \mathrm{C}-$ NMR $\left(50 \mathrm{MHz}, \mathrm{CDCl}_{3}\right): \delta=13.3\left(\mathrm{CH}_{3}\right) ; 23.4\left(\mathrm{CH}_{3}\right) ; 58.8\left(\mathrm{CH}_{2}\right) ; 94.6(\mathrm{C}-3) ; 124.6(\mathrm{CH}-5)$; 128.3 (p-C); $129.0(o-\mathrm{CH}) ; 129.9(m-\mathrm{CH}) ; 132.6(i-\mathrm{C}) ; 149.8(\mathrm{C}-4)$. MS (EI 70eV): $\mathrm{m} / z(\%)=$ $267.0\left(\mathrm{M}^{+}\right)$. Anal. Calcd for $\mathrm{C}_{13} \mathrm{H}_{17} \mathrm{NO}_{3} \mathrm{~S}$ (267.3) $\mathrm{C}$ : 58.40, H 6.41, N 5.24; O 17.95; found C 58.82, H 6.45, N 5.49, O 18.00\%.

General synthetic procedure for the preparation of 2-arylisothiazol-3(2H)-one 1,1-dioxides (10). The new sultams $\mathbf{1 0 b}, \mathbf{d}-\mathbf{g}$ are prepared according to the procedure described. ${ }^{10}$ 
4-Methyl-2-(4-methylphenyl)-isothiazol-3(2H)-one 1,1-dioxide (10b). Yield: 33\%, 0.07g. colourless needles. m.p. 200-202 ${ }^{\circ} \mathrm{C}$. IR (KBr-pellets, $\left.v_{\max }, \mathrm{cm}^{-1}\right): 1188\left(\mathrm{SO}_{2}\right), 1329\left(\mathrm{SO}_{2}\right), 1743$ $(\mathrm{C}=\mathrm{O}) .{ }^{1} \mathrm{H}-\mathrm{NMR}\left(200 \mathrm{MHz}, \mathrm{CDCl}_{3}\right): \delta=2.22\left(\mathrm{~d},{ }^{4} \mathrm{~J}=1.5 \mathrm{~Hz}, 3 \mathrm{H}, \mathrm{CH}_{3}\right) ; 2.41\left(\mathrm{~s}, 3 \mathrm{H}, p-\mathrm{CH}_{3}\right)$; $7.16\left(\mathrm{q},{ }^{4} \mathrm{~J}=1.5 \mathrm{~Hz}, 1 \mathrm{H},=\mathrm{CH}-5\right) ; 7.38-7.39$ (m, 4H, arom. H). ${ }^{13} \mathrm{C}-\mathrm{NMR}\left(50 \mathrm{MHz}, \mathrm{CDCl}_{3}\right): \delta=$ $12.5\left(\mathrm{CH}_{3}\right) ; 21.9\left(p-\mathrm{CH}_{3}\right) ; 126.5(i-\mathrm{C}) ; 128.7(o-\mathrm{CH}) ; 131.2(m-\mathrm{CH}) ; 132.1(\mathrm{CH}-5) ; 141.0(\mathrm{C}-4)$; $141.4(\mathrm{p}-\mathrm{C}) ; 160.8(\mathrm{C}=\mathrm{O})$. MS (EI 70eV): $\mathrm{m} / z(\%)=237.0\left(\mathrm{M}^{+}\right)$. Anal. Calcd for $\mathrm{C}_{11} \mathrm{H}_{11} \mathrm{NO}_{3} \mathrm{~S}$ (237.3) C: 55.68, H 4.67, N 5.90; O 20.23; found C 55.04, H 4.88, N 6.16, O 20.40\%.

2-(4-Chlorophenyl)-4-methyl-isothiazol-3(2H)-one 1,1-dioxide (10d). Yield: 10\%, 0.02g. colourless powder. m.p. 161-163 ${ }^{\circ} \mathrm{C}$. IR (KBr-pellets, $\left.v_{\max }, \mathrm{cm}^{-1}\right): 1187\left(\mathrm{SO}_{2}\right), 1331\left(\mathrm{SO}_{2}\right), 1743$ $(\mathrm{C}=\mathrm{O}) .{ }^{1} \mathrm{H}-\mathrm{NMR}\left(200 \mathrm{MHz}, \mathrm{CDCl}_{3}\right): \delta=2.23\left(\mathrm{~d},{ }^{4} \mathrm{~J}=1.5 \mathrm{~Hz}, 3 \mathrm{H}, \mathrm{CH}_{3}\right) ; 7.18\left(\mathrm{q},{ }^{4} \mathrm{~J}=1.5 \mathrm{~Hz}, 1 \mathrm{H}\right.$, $=\mathrm{CH}-5) ; 7.40\left(\mathrm{~d}, J_{\mathrm{AB}}=9.0 \mathrm{~Hz}, 2 \mathrm{H}\right.$, arom. H); $7.50\left(\mathrm{~d}, J=9.0 \mathrm{~Hz}, 2 \mathrm{H}\right.$, arom. H). ${ }^{13} \mathrm{C}-\mathrm{NMR}$ $\left(50 \mathrm{MHz}, \mathrm{CDCl}_{3}\right): \delta=12.5\left(\mathrm{CH}_{3}\right) ; 128.0(i-\mathrm{C}) ; 129.8(o-\mathrm{CH}) ; 130.9(\mathrm{~m}-\mathrm{CH}) ; 132.3(\mathrm{CH}-5)$; 136.7 ( $p$-C); $141.4(\mathrm{C}-4) ; 160.5(\mathrm{C}=\mathrm{O})$. MS (EI 70eV): $\mathrm{m} / z(\%)=257.0 / 259.0\left(\mathrm{M}^{+}\right)$. Anal. Calcd

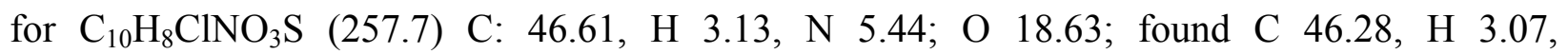
N 5.48, O $18.70 \%$.

2-(4-Bromophenyl)-4-methyl-isothiazol-3(2H)-one 1,1-dioxide (10e). Yield: 16\%, 0.04g. colourless crystals. m.p. $121-123^{\circ} \mathrm{C}$. IR (KBr-pellets, $\left.v_{\max }, \mathrm{cm}^{-1}\right): 1187\left(\mathrm{SO}_{2}\right), 1332\left(\mathrm{SO}_{2}\right), 1743$ $(\mathrm{C}=\mathrm{O}) .{ }^{1} \mathrm{H}-\mathrm{NMR}\left(200 \mathrm{MHz}, \mathrm{CDCl}_{3}\right): \delta=2.22\left(\mathrm{~d},{ }^{4} \mathrm{~J}=1.7 \mathrm{~Hz}, 3 \mathrm{H}, \mathrm{CH}_{3}\right) ; 7.18\left(\mathrm{q},{ }^{4} \mathrm{~J}=1.7 \mathrm{~Hz}, 1 \mathrm{H}\right.$, $=\mathrm{CH}-5) ; 7.34,\left(\mathrm{~d}, J_{\mathrm{AB}}=8.7 \mathrm{~Hz}, 2 \mathrm{H}\right.$, arom. $\left.\mathrm{H}\right) ; 7.64\left(\mathrm{~d}, J=8.7 \mathrm{~Hz}, 2 \mathrm{H}\right.$, arom. H). ${ }^{13} \mathrm{C}-\mathrm{NMR}$ $\left(50 \mathrm{MHz}, \mathrm{CDCl}_{3}\right): \delta=12.3\left(\mathrm{CH}_{3}\right) ; 124.6(p-\mathrm{C}) ; 128.4(i-\mathrm{C}) ; 129.8(o-\mathrm{CH}) ; 132.0(\mathrm{CH}-5)$; $133.6(m-\mathrm{CH}) ; 141.2(\mathrm{C}-4) ; 160.3(\mathrm{C}=\mathrm{O})$. MS (EI 70eV): $m / z(\%)=301.0 / 303.0\left(\mathrm{M}^{+}\right)$. Anal. Calcd for $\mathrm{C}_{10} \mathrm{H}_{8} \mathrm{BrNO}_{3} \mathrm{~S}$ (302.1) C: 39.75, H 2.67, N 4.64; O 15.89; found C 39.56, H 2.44, $\mathrm{N} 4.42$, O $16.00 \%$.

4-Ethyl-2-(4-methoxylphenyl)-isothiazol-3(2H)-one 1,1-dioxide (10f). Yield: 9\%, 0.02g. colourless needles. m.p. $133-134^{\circ} \mathrm{C}$. IR (KBr-pellets, $\left.v_{\max }, \mathrm{cm}^{-1}\right): 1180\left(\mathrm{SO}_{2}\right), 1256\left(\mathrm{OCH}_{3}\right)$, $1324\left(\mathrm{SO}_{2}\right), 1738(\mathrm{C}=\mathrm{O}) .{ }^{1} \mathrm{H}-\mathrm{NMR}\left(200 \mathrm{MHz}, \mathrm{CDCl}_{3}\right): \delta=1.22\left(\mathrm{t},{ }^{3} J=7.6 \mathrm{~Hz}, 3 \mathrm{H}, \mathrm{CH}_{3}\right) ; 2.61$ $\left(\mathrm{dq},{ }^{3} J=7.6 \mathrm{~Hz}, J=1.8 \mathrm{~Hz}, 2 \mathrm{H}, \mathrm{CH}_{2}\right) ; 3.84\left(\mathrm{~s}, 3 \mathrm{H}, \mathrm{OCH}_{3}\right) ; 7.01$ (d, $J=8.9 \mathrm{~Hz}, 2 \mathrm{H}$, arom. H), $7.09\left(\mathrm{~d},{ }^{3} J=1.8 \mathrm{~Hz}, 1 \mathrm{H},=\mathrm{CH}-5\right) ; 7.34\left(\mathrm{~d}, J_{\mathrm{AB}}=8.9 \mathrm{~Hz}, 2 \mathrm{H}\right.$, arom. H); ${ }^{13} \mathrm{C}-\mathrm{NMR}(50 \mathrm{MHz}$, $\left.\mathrm{CDCl}_{3}\right): \delta=11.6\left(\mathrm{CH}_{3}\right) ; 20.2\left(\mathrm{CH}_{2}\right) ; 56.2\left(\mathrm{OCH}_{3}\right) ; 115.9(m-\mathrm{CH}) ; 121.2(i-\mathrm{C}) ; 130.6(o-\mathrm{CH})$; 130.8 (CH-5); 147.3 (C-4); $160.6(\mathrm{p}-\mathrm{C}) ; 160.8(\mathrm{C}=\mathrm{O})$. MS (EI 70eV): $m / z(\%)=267.0\left(\mathrm{M}^{+}\right)$. Anal. Calcd for $\mathrm{C}_{12} \mathrm{H}_{13} \mathrm{NO}_{4} \mathrm{~S}$ (267.3) C: 53.92, $\mathrm{H} 4.90, \mathrm{~N} 5.24$; O 23.94; found $\mathrm{C}$ 54.10, $\mathrm{H} 4.85$, N 5.32, O 23.50\%.

4-Ethyl-2-phenyl-isothiazol-3(2H)-one 1,1-dioxide (10g). Yield: 21\%, 0.05g. colourless needles. m.p. $135-137^{\circ} \mathrm{C}$. IR (KBr-pellets, $\left.v_{\max }, \mathrm{cm}^{-1}\right)$ : $1182\left(\mathrm{SO}_{2}\right), 1322\left(\mathrm{SO}_{2}\right), 1739(\mathrm{C}=\mathrm{O})$. ${ }^{1} \mathrm{H}-\mathrm{NMR}\left(200 \mathrm{MHz}, \mathrm{CDCl}_{3}\right): \delta=1.27\left(\mathrm{t},{ }^{3} \mathrm{~J}=7.2 \mathrm{~Hz}, 3 \mathrm{H}, \mathrm{CH}_{3}\right) ; 2.61\left(\mathrm{dq},{ }^{3} \mathrm{~J}=7.2 \mathrm{~Hz},{ }^{4} \mathrm{~J}=2.0\right.$ $\left.\mathrm{Hz}, 2 \mathrm{H}, \mathrm{CH}_{2}\right) ; 7.1$ ( t $\left.{ }^{4} \mathrm{~J}=2,0 \mathrm{~Hz}, 1 \mathrm{H},=\mathrm{CH}-5\right) ; 7.43-7.54\left(\mathrm{~m}, 5 \mathrm{H}\right.$, arom. H). ${ }^{13} \mathrm{C}-\mathrm{NMR}(50 \mathrm{MHz}$, $\left.\mathrm{CDCl}_{3}\right): \delta=11.5\left(\mathrm{CH}_{3}\right) ; 20.2\left(\mathrm{CH}_{2}\right) ; 128.7(o-\mathrm{CH}) ; 129.4(p-\mathrm{CH}) ; 130.5(m-\mathrm{CH}) ; 130.8(\mathrm{CH}-5)$; $147.2(\mathrm{C}-4) ; 160.4(\mathrm{C}=\mathrm{O})$. MS (EI 70eV): $m / z(\%)=237.0\left(\mathrm{M}^{+}\right)$. Anal. Calcd for $\mathrm{C}_{11} \mathrm{H}_{11} \mathrm{NO}_{3} \mathrm{~S}$ (237.3) C: 55.68, H 4.67, N 5.90; O 20.23; found C 55.53, H 4.57, N 5.92, O 20.70\%. 
General procedure for the preparation of 2-aryl-3-methylene-2,3-dihydroisothiazole 1,1-dioxides (11). $1.0 \mathrm{mmol}$ 2-aryl-3-hydroperoxy-2,3-dihydroisothiazole 1,1-dioxide 7 is suspended in $3 \mathrm{~mL}$ distilled water and $1.0 \mathrm{mmol} \mathrm{Na} \mathrm{SO}_{3} \cdot 7 \mathrm{H}_{2} \mathrm{O}$ is added. After $24 \mathrm{hrs}$ stirring at room temperature $3 \mathrm{~mL}$ water are added to the mixture and extracted with $20 \mathrm{~mL}$ diethylether. The organic layers are washed with sat. NaCl-solution and water and dried over anhydrous $\mathrm{Na}_{2} \mathrm{SO}_{4}$. After evaporation of the solvent crystals of $\mathbf{1 1}$ are filtered off and dried.

2-(4-Methoxylphenyl)-4-methyl-3-methylene-2,3-dihydroisothiazole 1,1-dioxide (11a). Yield: $44 \%, 0.11 \mathrm{~g}$. colourless crystals. m.p. $108-110^{\circ} \mathrm{C}$. IR (KBr-pellets, $\left.v_{\max }, \mathrm{cm}^{-1}\right): 1139\left(\mathrm{SO}_{2}\right)$, $1252\left(\mathrm{OCH}_{3}\right), 1267\left(\mathrm{SO}_{2}\right) .{ }^{1} \mathrm{H}-\mathrm{NMR}\left(200 \mathrm{MHz}, \mathrm{CDCl}_{3}\right): \delta=2.17\left(\mathrm{~d},{ }^{4} \mathrm{~J}=1.4 \mathrm{~Hz}, 3 \mathrm{H}, \mathrm{CH}_{3}\right)$; $3.84\left(\mathrm{~s}, 3 \mathrm{H}, \mathrm{OCH}_{3}\right) ; 4.26\left(\mathrm{~m}, 1 \mathrm{H},=\mathrm{CH}_{2}{ }^{\mathrm{a}}\right) ; 4.63\left(\mathrm{~m}, 1 \mathrm{H},=\mathrm{CH}_{2}{ }^{\mathrm{b}}\right) ; 6.51(\mathrm{~s}(\mathrm{br}), 1 \mathrm{H},=\mathrm{CH}-5)$; $6.99\left(\mathrm{~d}, J=8.9 \mathrm{~Hz}, 2 \mathrm{H}\right.$, arom. H); 7.35 (d, $J=8.9 \mathrm{~Hz}, 2 \mathrm{H}$, arom. H). ${ }^{13} \mathrm{C}-\mathrm{NMR}(50 \mathrm{MHz}$, $\left.\mathrm{CDCl}_{3}\right): \delta=14.6\left(\mathrm{CH}_{3}\right) ; 57.0\left(\mathrm{OCH}_{3}\right) ; 92.2\left(\mathrm{CH}_{2}\right) ; 116.8(m-\mathrm{CH}) ; 122.2(\mathrm{CH}-5) ; 125.4(i-\mathrm{C})$; $133.2(o-\mathrm{CH}) ; 142.3(\mathrm{C}-4) ; 145.3(\mathrm{C}-3) ; 161.9(p-\mathrm{C})$. MS (EI 70eV): $m / z(\%)=251.0\left(\mathrm{M}^{+}\right)$. Anal. Calcd for $\mathrm{C}_{12} \mathrm{H}_{13} \mathrm{NO}_{3} \mathrm{~S}$ (251.3) C: 57.35, H 5.21, N 5.57; O 19.10; found C 56.86, H 5.42, N 5.51, O 19.00\%.

2-(4-Methylphenyl)-4-methyl-3-methylene-2,3-dihydroisothiazole 1,1-dioxide (11b). Yield: $63 \%, 0.15 \mathrm{~g}$. colourless crystals. m.p. $116-118^{\circ} \mathrm{C}$. IR (KBr-pellets, $\left.v_{\max }, \mathrm{cm}^{-1}\right): 1145\left(\mathrm{SO}_{2}\right)$, $1275\left(\mathrm{SO}_{2}\right) .{ }^{1} \mathrm{H}-\mathrm{NMR}\left(200 \mathrm{MHz}, \mathrm{CDCl}_{3}\right): \delta=2.20\left(\mathrm{~d},{ }^{4} \mathrm{~J}=1.3 \mathrm{~Hz}, 3 \mathrm{H}, \mathrm{CH}_{3}\right) ; 2.40(\mathrm{~s}, 3 \mathrm{H}$, $\left.p-\mathrm{CH}_{3}\right) ; 4.31\left(\mathrm{~m}, 1 \mathrm{H},=\mathrm{CH}_{2}{ }^{\mathrm{a}}\right) ; 4.65\left(\mathrm{~m}, 1 \mathrm{H}, \mathrm{CH}_{2}{ }^{\mathrm{b}}\right) ; 6.51(\mathrm{~s}(\mathrm{br}), 1 \mathrm{H},=\mathrm{CH}-5) ; 7.30-7.31(\mathrm{~m}, 4 \mathrm{H}$, arom. H). ${ }^{13} \mathrm{C}-\mathrm{NMR}\left(50 \mathrm{MHz}, \mathrm{CDCl}_{3}\right): \delta=13.4\left(\mathrm{CH}_{3}\right) ; 21.7\left(p-\mathrm{CH}_{3}\right) ; 91.3\left(\mathrm{CH}_{2}\right) ; 121.2(\mathrm{CH}-5)$; $128.5(o-\mathrm{CH}) ; 130.0(i-\mathrm{C}) ; 131.1(m-\mathrm{CH}) ; 140.0(\mathrm{C}-4) ; 140.8(p-\mathrm{C}) ; 143.8$ (C-3). MS (EI 70eV): $m / z(\%)=235.0\left(\mathrm{M}^{+}\right)$. Anal. Calcd for $\mathrm{C}_{12} \mathrm{H}_{13} \mathrm{NO}_{2} \mathrm{~S}$ (235.3) C: 61.25, H 5.57, N 5.95; O 13.60; found C 61.60, H 5.63, N 5.86, O 13.69\%.

4-Methyl-3-methylene-2-phenyl-2,3-dihydroisothiazole 1,1-dioxide (11c). Yield: 55\%, 0.12g. colourless crystals. m.p. $138-140^{\circ} \mathrm{C}$. IR (KBr-pellets, $\left.v_{\max }, \mathrm{cm}^{-1}\right): 1140\left(\mathrm{SO}_{2}\right), 1269\left(\mathrm{SO}_{2}\right)$. ${ }^{1} \mathrm{H}-\mathrm{NMR}\left(200 \mathrm{MHz}, \mathrm{CDCl}_{3}\right): \delta=2.18\left(\mathrm{~d},{ }^{4} \mathrm{~J}=1.3 \mathrm{~Hz}, 3 \mathrm{H}, \mathrm{CH}_{3}\right) ; 4.33\left(\mathrm{~m}, 1 \mathrm{H},=\mathrm{CH}_{2}{ }^{\mathrm{a}}\right)$; $4.68\left(\mathrm{~m}, 1 \mathrm{H}, \mathrm{CH}_{2}{ }^{\mathrm{b}}\right) ; 6.52(\mathrm{~s}(\mathrm{br}), 1 \mathrm{H},=\mathrm{CH}-5) ; 7.44-7.50\left(\mathrm{~m}, 5 \mathrm{H}\right.$, arom. H). ${ }^{13} \mathrm{C}-\mathrm{NMR}(50 \mathrm{MHz}$, $\left.\mathrm{CDCl}_{3}\right): \delta=13.8\left(\mathrm{CH}_{3}\right) ; 91.7\left(\mathrm{CH}_{2}\right) ; 121.3(\mathrm{CH}-5) ; 130.0(o-\mathrm{CH}) ; 130.4(m-\mathrm{CH}) ; 130.6(p-\mathrm{CH})$; $132.6(i-\mathrm{C}) ; 141.5(\mathrm{C}-4) ; 143.8(\mathrm{C}-3)$. MS (EI 70eV): $m / z(\%)=221.0\left(\mathrm{M}^{+}\right)$. Anal. Calcd for $\mathrm{C}_{11} \mathrm{H}_{11} \mathrm{NO}_{2} \mathrm{~S}$ (221.3) $\mathrm{C}: 59.71, \mathrm{H}$ 5.01, N 6.33; O 14.46; found $\mathrm{C} 60.06, \mathrm{H}$ 5.11, N 6.41, O $14.36 \%$.

2-(4-Chlorophenyl)-4-methyl-3-methylene-2,3-dihydroisothiazole 1,1-dioxide (11d). Yield: $10 \%, 0.02 \mathrm{~g}(1: 3$ mixture with $7 \mathbf{d})$. colourless crystals. ${ }^{1} \mathrm{H}-\mathrm{NMR}\left(200 \mathrm{MHz}, \mathrm{CDCl}_{3}\right): \delta=2.21$ $\left(\mathrm{d},{ }^{4} \mathrm{~J}=1.4 \mathrm{~Hz}, 3 \mathrm{H}, \mathrm{CH}_{3}\right) ; 4.36\left(\mathrm{~m}, 1 \mathrm{H},=\mathrm{CH}_{2}{ }^{\mathrm{a}}\right) ; 4.66\left(\mathrm{~d}, 1 \mathrm{H},=\mathrm{CH}_{2}{ }^{\mathrm{b}}\right) ; 6.50(\mathrm{~s}(\mathrm{br}), 1 \mathrm{H},=\mathrm{CH}-5)$; $7.51,8.17\left(\mathrm{~d}, 4 \mathrm{H}, J_{\mathrm{AB}}=9.2 \mathrm{~Hz}\right) .{ }^{13} \mathrm{C}-\mathrm{NMR}\left(50 \mathrm{MHz}, \mathrm{CDCl}_{3}\right): \delta=13.5\left(\mathrm{CH}_{3}\right) ; 91.9\left(\mathrm{CH}_{2}\right)$; 121.0 (CH-5); 124.5 (o-CH); $130.6(m-\mathrm{CH}) ; 130.8$ (p-C); 135.7 (i-C); 141.3 (C-4 ); 143.4 (C-3). MS (EI 70eV): $m / z(\%)=255.0 / 257.0\left(\mathrm{M}^{+}\right) . \mathrm{C}_{11} \mathrm{H}_{10} \mathrm{ClNO}_{2} \mathrm{~S}(255.7)$.

2-(4-Bromophenyl)-4-methyl-3-methylene-2,3-dihydroisothiazole 1,1-dioxide (11e). Yield: $12 \%, 0.03 \mathrm{~g}(2: 1 \mathrm{mixture}$ with $7 \mathrm{e})$. colourless crystals. ${ }^{1} \mathrm{H}-\mathrm{NMR}\left(200 \mathrm{MHz}, \mathrm{CDCl}_{3}\right): \delta=2.18$ $\left(\mathrm{d},{ }^{4} \mathrm{~J}=1.2 \mathrm{~Hz}, 3 \mathrm{H}, \mathrm{CH}_{3}\right) ; 4.34\left(\mathrm{~m}, 1 \mathrm{H},=\mathrm{CH}_{2}{ }^{\mathrm{a}}\right) ; 4.70\left(\mathrm{~m}, 1 \mathrm{H}, \mathrm{CH}_{2}{ }^{\mathrm{b}}\right) ; 6.52(\mathrm{~s}(\mathrm{br}), 1 \mathrm{H},=\mathrm{CH}-5)$; 
7.32-7.62 (d, 4H, $\left.J_{\mathrm{AB}}=8.6 \mathrm{~Hz}\right) .{ }^{13} \mathrm{C}-\mathrm{NMR}\left(50 \mathrm{MHz}, \mathrm{CDCl}_{3}\right): \delta=13.8\left(\mathrm{CH}_{3}\right) ; 92.0\left(\mathrm{CH}_{2}\right)$; 121.3 (CH-5); 129.9 (i-C); 131.9 (o-CH); 133.7 (p-C); 133.9 (m-CH); 142.8 (C-4 ); 143.6 (C-3). MS (EI 70eV): $m / z(\%)=299.0 / 301.0\left(\mathrm{M}^{+}\right) . \mathrm{C}_{11} \mathrm{H}_{10} \mathrm{BrNO}_{2} \mathrm{~S}(300.2)$.

4-Ethyl-3-methylene-2-phenyl-2,3-dihydroisothiazole 1,1-dioxide (11g). Yield: 86\%, 0.20g. colourless needles. m.p. $112-115^{\circ} \mathrm{C}$. IR (KBr-pellets, $\left.v_{\max }, \mathrm{cm}^{-1}\right): 1130\left(\mathrm{SO}_{2}\right), 1279\left(\mathrm{SO}_{2}\right)$. ${ }^{1} \mathrm{H}-\mathrm{NMR}\left(200 \mathrm{MHz}, \mathrm{CDCl}_{3}\right): \delta=1.28\left(\mathrm{t},{ }^{3} \mathrm{~J}=7.4 \mathrm{~Hz}, 3 \mathrm{H}, \mathrm{CH}_{3}\right) ; 2.54\left(\mathrm{dq},{ }^{3} J=7.4 \mathrm{~Hz}\right.$, $\left.{ }^{4} J=1.4 \mathrm{~Hz}, 2 \mathrm{H}, \mathrm{CH}_{2}\right) ; 4.31\left(\mathrm{t}, 1 \mathrm{H},=\mathrm{CH}_{2}{ }^{\mathrm{a}}\right) ; 4.69\left(\mathrm{~d}, 1 \mathrm{H}, \mathrm{CH}_{2}{ }^{\mathrm{b}}\right) ; 6.50(\mathrm{~s}(\mathrm{br}), 1 \mathrm{H},=\mathrm{CH}-5)$; 7.42-7.53 (m, 5H, arom. H). ${ }^{13} \mathrm{C}-\mathrm{NMR}\left(50 \mathrm{MHz}, \mathrm{CDCl}_{3}\right): \delta=11.6\left(\mathrm{CH}_{3}\right) ; 20.6\left(\mathrm{CH}_{2}\right)$; $91.2\left(\mathrm{CH}_{2}\right) ; 121.3(\mathrm{CH}-5) ; 130.4(o-\mathrm{CH}) ; 130.6(m-\mathrm{CH}) ; 132.0(p-\mathrm{CH}) ; 132.5(i-\mathrm{C}) ; 143.3(\mathrm{C}-4)$; $147.4(\mathrm{C}-3)$. MS (EI 70eV): $m / z(\%)=235.0\left(\mathrm{M}^{+}\right)$. Anal. Calcd for $\mathrm{C}_{12} \mathrm{H}_{13} \mathrm{NO}_{2} \mathrm{~S}(235.3) \mathrm{C}$ : 61.25, H 5.57, N 5.95; O 13.60; found C 61.50, H 5.61, N 5.84, O 13.66\%.

\section{References}

1. (a) Marco, J. L.; Ingate, S. T. Tetrahedron Lett. 1997, 38, 4835. (b) Ingate, S. T.; Marco, J. L.; Witvrouw, M.; Pannecouque, C.; DeClerq, E. Tetrahedron 1997, 53, 17795.

2. (a) Marco, J. L.; Ingate, S. T. Tetrahedron Lett. 1998, 39, 4123. (b) Marco, J. L.; Ingate, S. T.; Chinon, P. M. Tetrahedron 1999, 55, 7625.

3. Marco, J. L.; Ingate, S. T.; Jaime, C.; Bea, J. Tetrahedron 2000, 56, 23.

4. Fischer, R.; Kretschik, O.; Schenke, T.; Schenkel, R.; Wiedemann, J.; Erdelen, C.; Loesel, P.; Drewes, M. W.; Feucht, D; Andersch, W. W. Ger. Offen. DE 1999244 668, 1999; Chem. Abstr. 2001, 134, 4932.

5. Hanson, P. R.; Probst, D. A.; Robinson, R. E.; Yan, M. Tetrahedron Lett. 1999, 40, 4761.

6. Ho, K. F.; Fung, D. C. W.; Wong, W. Y.; Chan, W. H.; Lee, A. W. Tetrahedron Lett. 2001, 42,3121 .

7. Wanner, J.; Harmed A. M.; Probst, D. A.; Poon, K. W. C.; Klein, T. A.; Snelgrove, K. A.; Hanson, P. R. Tetrahedron Lett. 2002, 43, 917.

8. Taubert, K.; Sieler, J.; Hennig, L.; Findeisen, M.; Schulze, B. Helv. Chim. Acta 2002, 85, 183.

9. Taubert, K.; Siegemund, A.; Eilfeld, A.; Baumann, S.; Sieler, J.; Schulze, B. Synthesis 2003, $14,2265$.

10. Noack, A.; Jelonek, S.; Somoza Jr., F. B.; Schulze, B. J. Prakt. Chem. 1998, 340, 361.

11. Unterhalt, B.; Moghaddon, S. Pharm. 1994, 49, 317.

12. Schulze, B.; Obst, U.; Zahn, G.; Friedrich, B.; Cimiraglia, R.; Hofmann H.-J. J. Prakt. Chem. 1995, 337, 175.

13. Schulze, B.; Rosenbaum, K.; Hilbig, J.; Weber, L. J. Prakt. Chem. 1992, 334, 25. 